\title{
MicroRNA-29b Inhibits Angiogenesis by Targeting VEGFA through the MAPK/ ERK and PI3K/Akt Signaling Pathways in Endometrial Carcinoma
}

\author{
Hong-Xia Chen ${ }^{a, b}$ Xiao-Xia Xu $\quad$ Zhi Zhang ${ }^{b}$ Bu-Zhen Tan ${ }^{a}$ Xiao-Dong Zhou \\ aDepartment of Obstetrics and Gynecology, The Second Affiliated Hospital of Nanchang University, \\ Nanchang, bepartment of Obstetrics and Gynecology, The First Affiliated Hospital of Nanchang \\ University, Nanchang, 'Department of Gastroenterology, The First Affiliated Hospital of Nanchang \\ University, Nanchang, China
}

\section{Key Words}

MicroRNA-29b • Endometrial carcinoma - VEGFA - MAPK/ERK signaling pathway • PI3K/Akt signaling pathway $\cdot$ Angiogenesis

\begin{abstract}
Objective: The purpose of this study is to explore the effects of microRNA-29b (miR-29b) regulating MAPK/ERK and PI3K/Akt signaling pathways on angiogenesis in endometrial carcinoma (EC) by targeting VEGFA. Methods: Between February 2013 and April 2015, 126 EC patients admitted to the Second Affiliated Hospital of Nanchang University were randomly selected, with $126 \mathrm{EC}$ tissues and the corresponding adjacent normal tissues collected after surgery. The human EC cell lines RL-95-2 and HEC-1-B and human endometrial cells were assigned to the normal group (human endometrial cells), the blank group (untransfected RL95-2 or HEC-1-B cells), the pMIR-control group (RL-95-2 or HEC-1-B cells transfected with an empty vector), the pMIR-miR-29b group (RL-95-2 or HEC-1-B cells transfected with the miR29b plasmid), LNA-control group (RL-95-2 or HEC-1-B cells transfected with an oligonucleotide inhibitors control), the LNA-miR-29b inhibitors group (RL-95-2 or HEC-1-B cells transfected with miRCURY LNA ${ }^{\mathrm{TM}}$ miR-29b inhibitors), the LNA-miR-29b inhibitors + PD98059 group (RL95-2 or HEC-1-B cells transfected with miRCURY LNA ${ }^{\text {TM }}$ miR-29b inhibitors and PD98059, an inhibitor of the MAPK/ERK signaling pathway) and the LNA-miR-29b inhibitors + wortmannin group (RL-95-2 or HEC-1-B cells transfected with miRCURY LNA ${ }^{\text {TM }}$ miR-29b inhibitors and wortmannin, an inhibitor of the PI3K/Akt signaling pathway). QRT-PCR and Western blotting were conducted to detect the miR-29b expression and the MRNA and protein expressions of VEGFA, ERK, Akt, mTOR and Bcl-2. Immunohistochemistry (IHC) was performed to determine the microvessel density (MVD) expression in the EC tissues, adjacent normal tissues and nude-mice. Results: Compared with the adjacent normal tissues, miR-29b expression was down-regulated, the mRNA and protein expressions of VEGFA, ERK, Akt, mTOR and Bcl-2 were up-regulated, and MVD expression was increased in the EC tissues. Compared with the normal group, miR-29b expression was down-regulated, while the mRNA and protein


expressions of VEGFA, ERK, Akt, mTOR and Bcl-2 were up-regulated in the other groups. Compared with the blank, pMIR-control and LNA-control groups, miR-29b expression was increased, while mRNA and protein expressions of VEGFA, ERK, Akt, mTOR and Bcl-2 were decreased in the pMIR-miR-29b group. The LNA-miR-29b inhibitors group exhibited elevated miR-29b expression and decreased mRNA and protein expressions of VEGFA, ERK, Akt, mTOR and $\mathrm{BCl}-2$ (All $P<0.05$ ). Additionally, miR-29b expression was reduced in the LNA-miR-29b inhibitors + PD98059 and LNA-miR-29b inhibitors + wortmannin groups. In comparison to the normal group, MVD expression was elevated in the other groups. Compared with the blank, pMIR-control, LNA-control, LNA-miR-29b inhibitors + PD98059 and LNA-miR-29b inhibitors + wortmannin groups, MVD expression was decreased in the pMIR-miR-29b group but increased in the LNA-miR-29b inhibitors group. Conclusion: Our results indicate that miR-29b negatively modulates the MAPK/ERK and PI3K/Akt signaling pathways to inhibit angiogenesis in EC by targeting VEGFA.

\section{Introduction}

(C) 2017 The Author(s)

Published by S. Karger AG, Basel

Endometrial carcinoma (EC) is a significant cause of morbidity and mortality for women across the world [1]. EC in its early stages is often satisfactorily treated with surgery, but advanced EC is characterized by difficult treatment and a poor prognosis [2], especially in terms of recurrent or metastatic disease [3]. The 5-year survival rate of EC patients with localized disease exceeds $90 \%$, while this rate for patients with distant disease at diagnosis is below 20\% [4]. Current treatment regimens, including hormonal therapy, hysterectomy, and combinations of chemotherapy and radiation, are efficient for early-stage EC; however, therapies for advanced EC patients are unavailable, resulting in a dismal outcome [5]. Therefore, identification of additional cellular and molecular mechanisms responsible for the progression and tumorigenesis of EC and the discovery of new diagnostic and prognostic markers and novel therapeutic strategies for EC are critical for improving the clinical outcome of EC patients.

MicroRNAs (miRNAs) are approximately 0 22 nucleotide non-coding small RNAs that can function as specific sequence regulators of gene expression by translational repression and transcript cleavage $[6,7]$. MiRNAs modulate several cellular activities, including cell growth, cell differentiation, metabolic activities, apoptosis, angiogenesis, and tissue turnover [8]. The abnormal expression of miRNAs has been closely connected with the development and progression of various human diseases, specifically diseases associated with cellular transformation, inflammation, tumorigenesis, and tissue fibrosis [9-12]. For example, the overexpression of miR-10b in adenomyotic epithelial cells suppresses the cell migration and invasion [13]. MiRNA-29b (miR-29b) is an important member of the miRNA family [14] and has been demonstrated to be dysregulated in many tumors [15-17]. Particularly, it has been demonstrated that miR-29b can serve as a tumor suppressor that inhibits the proliferation process of cancer cells [18]. However, the molecular mechanisms through which the dysregulation of miR-29b contributes to the diagnosis and prognosis of EC remain to be further elucidated.

Angiogenesis refers to the formation of novel blood vessels from preexisting ones and plays a major role in tumor growth, development and progression, and metastasis [1]. Vascular endothelial growth factor A (VEGFA) is a pro-angiogenic factor involved in the proliferation, differentiation, and migration of endothelial cells and participates in vascular permeability [19]. In the uterus, it has been reported that estrogen can rapidly activate various signaling pathways, including the mitogen-activated protein kinase/extracellular regulated protein kinases (MAPK/ERK) and phosphatidylinositol 3-kinase/protein kinase $\mathrm{B}$ (PI3K/Akt) signaling pathways in the caveolae [20]. Given the former analyses, our study aims to investigate the role of miR-29b regulating the MAPK/ERK and PI3K/Akt signaling pathways in angiogenesis in EC by targeting VEGFA to seek novel insight into the treatment of EC. 


\section{Cellular Physiology Cell Physiol Biochem 2017;41:933-946 and Biochemistry Publisned \begin{tabular}{l|l} 
DOI: 10.1159/000460510 & $\begin{array}{l}\text { (c) } 2017 \text { The Author(s). Published by S. Karger AG, Basel } \\
\text { www.karger.com/cpb }\end{array}$
\end{tabular}}

Chen et al.: MiR-29b Inhibits Angiogenesis in EC

\section{Material and Methods}

Subjects

A total of 126 EC patients admitted to the Second Affiliated Hospital of Nanchang University between February 2013 and April 2015 were randomly recruited in our study, with 126 EC tissues and the corresponding adjacent normal tissues ( $2 \mathrm{~cm}$ away from the distal EC tissues) were collected after surgery. The clinicopathological characteristics of patients were also obtained. The type I (estrogen-dependent) EC cell line RL-95-2 and type II (estrogen-independent) EC cell line HEC-1-B were purchased from the Cell Bank of the Shanghai Institutes for Biological Sciences (Chinese Academy of Sciences, Shanghai, China). Human endometrial cells were purchased from Shanghai Biotechnology Co., Ltd. Enzyme Research (Shanghai, China). In addition, 160 3-month-old BALB/C nude mice (female, weighing $250 \pm 50 \mathrm{~g}$ ) were obtained from the Animal Laboratory Center of China Medical University (Shenyang, China). All animal experiments in the present study were in accordance with the Guide for the Care and Use of Laboratory Animals issued by the National Institutes of Health.

\section{Construction of miR-29b and LNA-miR-29b inhibitors plasmid vectors}

The precursor sequences of miR-29b were amplified, and primers were designed for polymerase chain reaction (PCR) amplification. Kpn I and EcoR I cleavage sites were added to the two ends of the primers. The sequences were synthesized by Shanghai GenePharma Co., Ltd. (Shanghai, China) (Table 1). A sequence showing no homology to the mammalian genome was selected as a negative control (NC) of the doublestranded RNA. The section was cloned into a pMIR lentivirus vector; thus, pMIR-miR-29b (miR-29b plasmid) and pMIR-vector (Empty plasmid) were formed correspondingly. The miRCURY LNA Power inhibitor was purchased from Exiqon A/S Inc. (Vedbaek, Denmark). The miR-29b oligonucleotide inhibitors of miRCURY LNA $^{\mathrm{TM}}$ miR-29b inhibitor (LNA-miR-29b inhibitors) were constructed, and at the same time, the miR-29b oligonucleotide inhibitors control (LNA-control) was set. The target gene segments of VEGFA were inserted into the wild type VEGFA-3'UTR-WT plasmid and mutant VEGFA-3'UTR-MUT plasmid to construct a dual luciferase reporter vector of VEGFA. The above plasmids were identified by PCR, enzyme digestion and sequencing.

\section{Cell transfection and grouping}

The human EC cell lines RL-95-2 and HEC-1-B and human endometrial cells at the logarithmic growth phase were seeded into 96-well plates (100 $\mu \mathrm{L} /$ well). The lentiviral transfection group: RL-95-2 cells and HEC-1-B cells were transfected by miR-29b lentivirus and control in $40 \%$ cell fusion. The morphology of the cells was observed 12 hours after the transfection, and then, the cells were cultured in normal media after 16 hours. Total RNA and protein from cells were extracted after 72 hours. The oligonucleotide inhibitors group: RL-95-2 cells and HEC-1-B cells were transfected by miRCURY LNA ${ }^{\mathrm{TM}}$ miR-126 inhibitor and control in 40\% cell fusion with Lipofectamine 2000. The cell morphology was observed 2 to 4 hours after the transfection, and then, cells were cultured in normal media after 6 hours. Total RNA and protein from cells were extracted after 72 hours. Th cell group design was as follows: the normal group (human endometrial cells), the blank group (untransfected RL-95-2 or HEC-1-B cells), the pMIR-control group (RL95-2 or HEC-1-B cells transfected with an empty vector), the pMIR-miR-29b group (RL-95-2 or HEC-1-B cells transfected with the miR-29b plasmid), LNA-control group (RL-95-2 or HEC-1-B cells transfected

Table 1. Oligonucleotide sequences of RNA and DNA. Notes: miR-29b, microRNA-29b; NC, negative control of double-stranded RNA; VEGFA, vascular endothelial growth factor $\mathrm{A}$

\begin{tabular}{ll}
\hline & Sequence \\
\hline miR-29b & Upstream: 5'-TGAACCTTTGTCTGGGCAACT-3' \\
& Downstream: 5'-TGGTATCCTTGAGGGATTGGTTC-3' \\
NC & 5'-UUCUCCGAACGUGUCACGUTT-3' \\
LNA-miR-29b inhibitor & 5'-ACTGATTTCAAATGGTGCT-3' \\
LNA-control & 5'-GTGTAACACGTCTATACGCCCA-3' \\
VEGFA & Upstream: 5'-GAGCCTTGCCTTGCTGCTCTA-3' \\
& Downstream: 5'-CACCAGGGTCTCGATTGGATG-3' \\
\hline
\end{tabular}




\section{Cellular Physiology Cell Physiol Biochem 2017;41:933-946

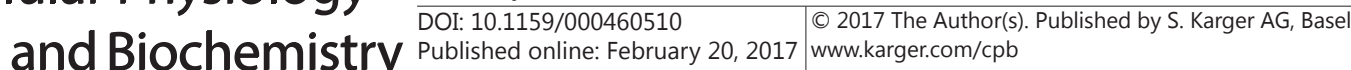 \\ Chen et al.: MiR-29b Inhibits Angiogenesis in EC}

with the oligonucleotide inhibitors control), the LNA-miR-29b inhibitors group (RL-95-2 or HEC-1-B cells transfected with miRCURY LNA ${ }^{\text {TM }}$ miR-29b inhibitors), the LNA-miR-29b inhibitors + PD98059 group (RL95-2 or HEC-1-B cells transfected with miRCURY LNA ${ }^{\mathrm{TM}}$ miR-29b inhibitors and PD98059, an inhibitor of the MAPK/ERK signaling pathway) and the LNA-miR-29b inhibitors + wortmannin group (RL-95-2 or HEC-1-B cells transfected with miRCURY LNA ${ }^{\mathrm{TM}}$ miR-29b inhibitors and wortmannin, an inhibitor of the PI3K/Akt signaling pathway).

\section{Tumor xenografts in nude mice and grouping}

RL-95-2 cells and HEC-1-B cells at the logarithmic growth phase in the above groups were adjusted to $1 \times 10^{6} / \mathrm{mL}$ and inoculated in right abdominal subcutaneous of BALB/C nude mice ( $1 \mathrm{~mL}$ per mouse). A total of 160 nude mice were classified into RL-95-2 mice $(n=80)$ and HEC-1-B mice $(n=80)$. The RL-95-2 mice and HEC-1-B mice were further divided into the normal group (nude mice without any treatment), the blank group (nude mice inoculated with RL-95-2 or HEC-1-B cells without any transfection), the pMIRcontrol group (nude mice inoculated with RL-95-2 or HEC-1-B cells transfected with an empty vector), the pMIR-miR-29b group (nude mice inoculated with RL-95-2 or HEC-1-B cells transfected with the miR-29b plasmid), the LNA-control group (nude mice inoculated with RL-95-2 or HEC-1-B cells transfected with the oligonucleotide inhibitor), LNA-miR-29b inhibitors group (nude mice inoculated with RL-95-2 or HEC1-B cells transfected with miRCURY LNA ${ }^{\mathrm{TM}}$ miR-29b inhibitors), LNA-miR-29b inhibitors + PD98059A group (nude mice inoculated with RL-95-2 or HEC-1-B cells transfected with miRCURY LNA ${ }^{\mathrm{TM}}$ miR-29b inhibitors and PD98059), LNA-miR-29b inhibitors + wortmannin group (nude mice inoculated with RL-95-2 or HEC1-B cells transfected with miRCURY LNA ${ }^{\mathrm{TM}}$ miR-29b inhibitors and wortmannin). There were 10 mice in each group. Then, all nude mice were killed by cervical dislocation. Subsequently, the infection of the inoculation site and tumor growth condition was observed every day. The long diameter (a) and short diameter (b) of tumors in nude mice were measured at the $1^{\text {st }}, 2^{\text {nd }}, 4^{\text {th }}$ and $6^{\text {th }}$ week. Tumor-growth curves were analyzed using the following tumor volume calculation formula: $\mathrm{V}=1 / 2 \mathrm{ab}^{2}$. The tumor in right abdominal subcutaneous of nude mice was dissected and weighed after the death of the nude mice, and the tumor control rate in each group was calculated using the formula as follows: tumor control rate $(\%)=\mathrm{V}$

$/ \mathrm{V}_{\text {blank group }} \times 100 \%$. Endometrial tumor metastasis was observed by laparotomy, and the average number of endometrial tumor metastatic nodules was calculated. Inhibition rate of endometrial nodule metastasis (\%) $=(1$-Average number of metastatic nodules in the treatment group/Average number of metastatic nodules in the blank group) $\times 100 \%$.

\section{Quantitative real-time polymerase chain reaction ( $q R T$-PCR)}

The EC tissues and adjacent normal tissues were ground in normal saline. The cells and total RNA in each transfection group were extracted using RNA reagent kit (Invitrogen Inc., Carlsbad, CA, USA). Primers of miR-29b, VEGFA, ERK, Akt, mTOR and B-cell lymphoma-2 (Bcl-2) were designed and synthetized by Shanghai Sangon Biological Engineering Technology \& Services Co., Ltd. (Shanghai, China) (Table 2). The total RNA was reversely transcribed into cDNA through the specific stem-loop reverse transcription primer in the TaqMan $\AA$ MicroRNA Reverse Transcription Kit. The reverse transcription system was conducted in $10 \mu \mathrm{L}$, and the reaction conditions were as follows: $16^{\circ} \mathrm{C}$ for $30 \mathrm{~min}, 42^{\circ} \mathrm{C}$ for $30 \mathrm{~min}$ and $85^{\circ} \mathrm{C}$ for $10 \mathrm{~min}$. The TaqMan ${ }^{\circledR}$ MicroRNA Assays was applied in the qRT-PCR, and the reaction conditions were as follows: 40 cycles of pre-denaturation for $3 \mathrm{~min}$ at $95^{\circ} \mathrm{C}$, denaturation for $15 \mathrm{~s}$ at $95^{\circ} \mathrm{C}$, annealing for $30 \mathrm{~s}$ at $60^{\circ} \mathrm{C}$, and extension for $30 \mathrm{~s}$ at $72^{\circ} \mathrm{C}$. The reaction system included $2 \mu \mathrm{L}$ PCR upstream primer, $2 \mu \mathrm{L}$ PCR downstream primer, $4 \mu \mathrm{L}$ DNA template, $1 \mu \mathrm{L}$ ROX Reference Dye $(50 \times)$, $25 \mu \mathrm{L}$ SYBR Premix Ex TaqII and $16 \mu \mathrm{L} \mathrm{dH}_{2} \mathrm{O}$. The relative expressions were calculated according to $2^{-\Delta \Delta \mathrm{Ct}}$, with $\mathrm{U} 6 / \beta$-actin as the internal control.

\section{Western blotting}

The EC tissues, adjacent normal tissues and cells in each transfection group were cracked, and the total protein was extracted. After mixing $20 \mu \mathrm{g}$ cell protein with the sample buffer, sodium dodecyl sulfatepolyacrylamide gel electrophoresis (SDS-PAGE) was performed. Proteins that separated by electrophoresis were transferred to a nitrocellulose filter, and 5\% skimmed milk powder-phosphate buffered saline (PBS) solution was added for 1 hour at room temperature and incubated overnight with VEGFA, ERK, Akt, mTOR and Bcl- 2 antibody at $4^{\circ} \mathrm{C}$. The filter membrane was washed with the PBS buffer 3 times and incubated with horseradish peroxidase (HRP) labeled with a secondary antibody for 1 hour at room temperature. Then, after being washed with PBS 3 times again, the filter membrane was exposed and shadowed by ECL light KARGER 
Table 2. Primer sequences for quantitative real-time polymerase chain reaction. Notes: miR-29b, microRNA-29b; VEGFA, vascular endothelial growth factor A; ERK, extracellular regulated protein kinases; Akt, protein kinase B; mTOR, mechanistic target of rapamycin; Bcl-2, B-cell lymphoma-2

\begin{tabular}{|c|c|}
\hline Gene & Sequence \\
\hline \multirow[t]{2}{*}{ miR-29b } & Upstream: 5'-GGGTAGCACCATTTGAAATC-3' \\
\hline & Downstream: 5'-TTTGGCACTAGCACATT-3' \\
\hline \multirow[t]{2}{*}{ VEGFA } & Upstream: 5'-TGCACCCACGACAGAAGGGGA-3' \\
\hline & Downstream: 5'-TCACCGCCTTGGCTTGCTACAT-3' \\
\hline \multirow[t]{2}{*}{ ERK } & Upstream: 5'-CCAGAGTGGCTATCAAGAAG-3' \\
\hline & Downstream: 5'-TCCATGAGGTCCTGAACAA-3' \\
\hline \multirow[t]{2}{*}{ Akt } & Upstream: 5'ATGAGCGACGTGGCTATTGTGAAG3' \\
\hline & Downstream: 5'GAGGCCGTCAGCCACAGTCTGGATG3' \\
\hline \multirow[t]{2}{*}{ mTOR } & Upstream: 5’-CTGGGACTCAAATGTGTGCAGTTC-3’ \\
\hline & Downstream: 5'-GAACAATAGGGTGAATGATCCGGG-3' \\
\hline \multirow[t]{2}{*}{$\mathrm{Bcl}-2$} & Upstream: 5’-ATCTTCTCCTTCCAGCCTGA-3’ \\
\hline & Downstream: 5'-TCAGTCATCCACAGAGCGAT-3' \\
\hline \multirow[t]{2}{*}{$\beta$-actin } & Upstream: 5’- GAATCCACTGGCGTCTTCAC -3' \\
\hline & Downstream: 5'- CGTTGCTGACAATCTTGAGAGA -3' \\
\hline
\end{tabular}

emitting liquid and shot by Bio-RAD Gel system (Bio-Rad, Inc., Hercules, CA, USA) for analysis of gray value. With glyceraldehyde-3-phosphate dehydrogenase (GAPDH) (Bioworld Technology, Inc., Atlanta, GA, USA) as a reference, the relative protein expression was described as the ratio of the gray value of the target band to the gray value of the reference band.

Immunohistochemistry (IHC)

The EC tissues, adjacent normal tissues and nude mouse EC tissues were embedded in paraffin wax, dissected, and baked for $20 \mathrm{~min}$ at $68^{\circ} \mathrm{C}$. The tissues were dewaxed in conventional xylene, alcohol dehydrated, placed at room temperature for $15 \mathrm{~min}$, and washed with the PBS solution 2-3 times/5 min and goat serum blocking fluid was added for $20 \mathrm{~min}$ at room temperature. The sections were incubated for one hour with the secondary antibody to CD34 (PL Laboratories Inc., British Columbia, Canada) at $37^{\circ} \mathrm{C}$ for 1 hour and finally washed with PBS. Diaminobenzidine (DAB) was used for coloration, hematoxylin was added to redye for $2 \mathrm{~min}$, and microscopy was ultimately performed after conventional dehydration, hyaline and mounting. The CD34 antibody was applied to label the vascular endothelial cells. Tan single endothelial cells or endothelial cells were counted as a blood vessel to determine the level of angiogenesis.

\section{Dual-luciferase reporter assay}

The targeting relationship between miR-29b and VEGFA was predicted by microRNA.org and validated by the dual luciferase reporter gene. The $293 \mathrm{~T}$ cells at the logarithmic growth phase were inoculated into 96-well plates. When the cell density reached $70 \%$, the cells were transfected with Lipofectamine 2000, and the VEGFA-3'UTR-WT and miRNA-29b plasmids were mixed with each other and co-transfected into the 293T cells. At the same time, VEGFA-3'UTR-WT + NC and VEGFA-3'UT R-MUT + miR-29b were set as the control. After $6 \mathrm{~h}$ of culture, the cells were cultured in a new medium containing $10 \%$ fetal bovine serum (FBS) for $48 \mathrm{~h}$. Then, chemiluminescence was conducted to detect enzymatic activity.

\section{Statistical analysis}

SPSS 21.0 statistical software (SPSS Inc., Chicago, IL, USA) was used for the data analysis. The measurement data were expressed using the mean \pm standard deviation. Comparisons between two groups were analyzed by $t$-test, and comparisons among groups were analyzed using one-way analysis of variance (ANOVA). In addition, $\chi^{2}$ test was used for comparisons of the count data among groups. $P<0.05$ was considered statistically significant.

\section{Results}

Clinicopathological characteristics of EC patients

Among the 126 EC patients (mean age: $51.2 \pm 10.5$ years), there were 81 cases with depth of myometrium invasion $<1 / 2$ and 45 cases with depth of myometrium invasion $>1 / 2$. None of these patients had a history of abortion. According to the International Federation of 
Gynecology and Obstetrics (FIGO) staging system [21], the patients were classified as stage I $(n=90)$, stage II $(n=15)$, stage III $(n=17)$ and stage IV $(n=4)$. Histologically, 47 cases were highly differentiated, 59 were moderately differentiated, and 20 were poorly differentiated. Regarding the pathological type, there were 100 cases with endometrioid adenocarcinoma and 26 cases with special types of EC. There were 18 cases with lymph node metastasis and 108 cases without lymph node metastasis. Among these patients, there were 84 cases with type I EC and 42 cases with type II EC (Table 3).

Comparisons of the expressions of miR-29b, VEGFA, ERK, Akt, mTOR and Bcl-2 between the EC and adjacent normal tissues

Compared with the adjacent normal tissues, miR-29b expression was down-regulated, while the mRNA expressions of VEGFA, ERK, Akt, mTOR and Bcl-2 were up-regulated in the EC tissues $(P<0.05)$ (Fig. 1), which suggested that miR-29b was negatively correlated with VEGFA, ERK, Akt, mTOR and Bcl-2. Additionally, the protein expressions of VEGFA, ERK, Akt, mTOR and Bcl-2 were elevated in the EC tissues compared with the adjacent normal tissues $(P<0.05)$ (Fig. 2), suggesting that the over-expression of VEGFA, ERK, Akt, mTOR and Bcl-2 were correlated with the progression of EC.

Correlations of miR-29b and VEGFA mRNA expressions with the clinicopathological characteristics in EC patients

The miR-29b expression was higher in stage I-II than in stage III-IV, while VEGFA mRNA expression was lower in stage I-II than in stage III-IV in EC patients (all $P<0.01$ ). The miR-29b expression of patients with lymph-node metastasis was lower, but the expression of VEGFA mRNA was higher, than those in patients without lymph-node metastasis (all $P<0.01$ ). The miR-29b and VEGFA mRNA expressions had no significant differences in the myometrium invasion, degree of differentiation and pathological types (all $P>0.05$ ) (Table 4).

Comparison of MVD expression between the EC and adjacent normal tissues

The MVD expression of the human EC tissues and adjacent normal tissues were 10.31 \pm 1.04 and $5.49 \pm 0.55$, respectively $(P<0.05)$ (Fig. 3).

Table 3. Clinicopathological characteristics of EC patients. Note: EC, endometrial carcinoma; FIGO, Federation of Gynecology and Obstetrics

\begin{tabular}{lll}
\hline Clinicopathological characteristic & $\begin{array}{l}\mathrm{I} \\
\mathrm{n}=84\end{array}$ & $\begin{array}{l}\mathrm{II} \\
\mathrm{n}=42\end{array}$ \\
\hline $\begin{array}{l}\text { Age (years) } \\
<55\end{array}$ & $58.30 \%$ & $54.80 \%$ \\
$>55$ & $41.70 \%$ & $45.20 \%$ \\
$\begin{array}{l}\text { Depth of myometrium invasion } \\
<1 / 2\end{array}$ & $67.90 \%$ & $57.10 \%$ \\
$>1 / 2$ & $32.10 \%$ & $42.90 \%$ \\
Histological differentiation & & \\
high differentiation & $34.50 \%$ & $42.90 \%$ \\
moderate differentiation & $56.00 \%$ & $28.60 \%$ \\
poor differentiation & $9.50 \%$ & $28.60 \%$ \\
FIGO stage & & \\
Stage I & $75.00 \%$ & $64.30 \%$ \\
Stage II & $9.50 \%$ & $16.70 \%$ \\
Stage III & $3.10 \%$ & $14.30 \%$ \\
Stage VI & $2.40 \%$ & $4.80 \%$ \\
Prognosis (5 years) & & \\
Survival rate & $>85 \%$ & $>80 \%$ \\
Recurrence rate & $<9 \%$ & $<17 \%$ \\
\hline
\end{tabular}




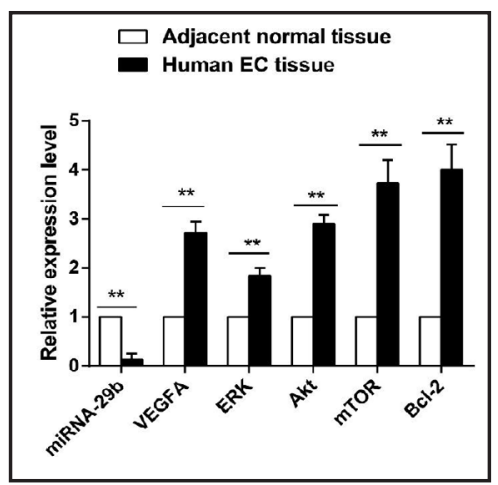

Fig. 1. The expression of miR-29b and the mRNA expressions of VEGFA, ERK, Akt, mTOR and Bcl-2 between the human EC tissues and adjacent normal tissues. EC, endometrial carcinoma; miR-29b, microRNA-29b; VEGFA, vascular endothelial growth factor A; ERK, extracellular regulated protein kinases; Akt, protein kinase B; mTOR, mammalian target of rapamycin; Bcl-2, B-cell lymphoma-2; *, $P<0.01$ in comparison to the adjacent normal tissues.

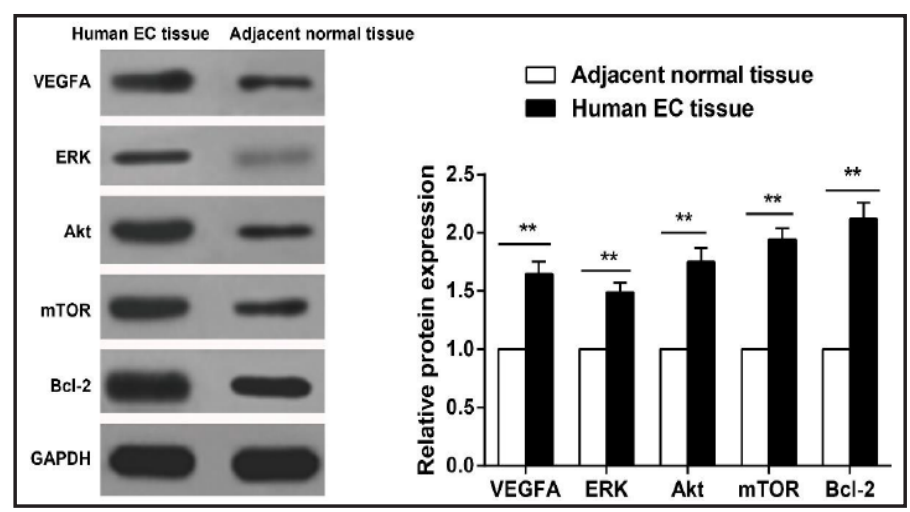

Fig. 2. Protein expressions of VEGFA, ERK, Akt, mTOR and Bcl-2 between the human EC tissues and adjacent normal tissues. EC, endometrial carcinoma; miR-29b, microRNA-29b; VEGFA, vascular endothelial growth factor A; ERK, extracellular regulated protein kinases; Akt, protein kinase B; mTOR, mammalian target of rapamycin; Bcl-2, B-cell lymphoma- 2 ; $^{*} P<0.01$ in comparison to the adjacent normal tissues.

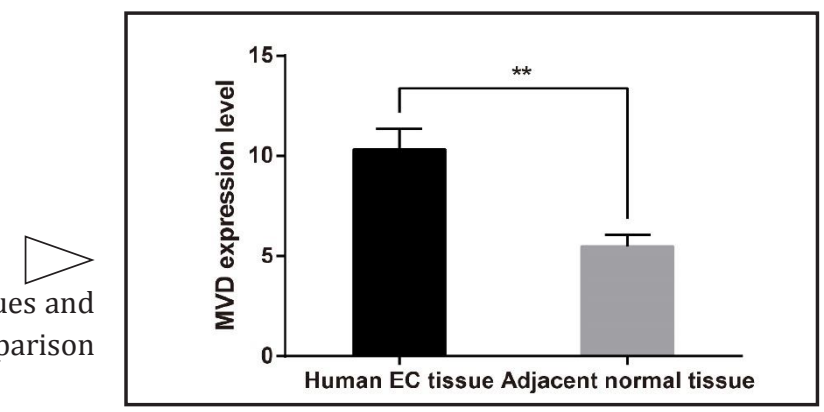

Table 4. Correlations of miR-29b and VEGFA mRNA expressions with clinicopathological characteristics in EC patients ( $\bar{x} \pm s$ ). Notes: miR-29b, microRNA-29b; VEGFA, vascular endothelial growth factor A; FIGO, Federation of Gynecology and Obstetrics

\begin{tabular}{|c|c|c|c|c|c|}
\hline Clinicopathological characteristic & $\mathrm{n}$ & miR-29b expression & $P$ & VEGFA expression & $P$ \\
\hline Depth of myometrium invasion & & & 0.865 & & 0.434 \\
\hline$<1 / 2$ & 81 & $0.833 \pm 0.177$ & & $0.303 \pm 0.062$ & \\
\hline$>1 / 2$ & 45 & $0.826 \pm 0.284$ & & $0.314 \pm 0.095$ & \\
\hline FIGO stage & & & 0.009 & & 0.001 \\
\hline I + II & 105 & $0.853 \pm 0.210$ & & $0.297 \pm 0.060$ & \\
\hline III + IV & 21 & $0.716 \pm 0.240$ & & $0.356 \pm 0.119$ & \\
\hline Histological differentiation & & & 0.642 & & 0.83 \\
\hline high differentiation & 47 & $0.843 \pm 0.166$ & & $0.305 \pm 0.068$ & \\
\hline moderate + poor differentiation & 79 & $0.824 \pm 0.248$ & & $0.308 \pm 0.080$ & \\
\hline Pathologic type & & & 0.637 & & 0.08 \\
\hline endometrial carcinoma & 100 & $0.835 \pm 0.197$ & & $0.301 \pm 0.058$ & \\
\hline special type & 26 & $0.812 \pm 0.296$ & & $0.330 \pm 0.120$ & \\
\hline Lymph node metastasis & & & $<0.001$ & & $<0.001$ \\
\hline No & 108 & $0.864 \pm 0.215$ & & $0.294 \pm 0.061$ & \\
\hline Yes & 18 & $0.623 \pm 0.134$ & & $0.386 \pm 0.102$ & \\
\hline
\end{tabular}

VEGFA as a target gene of miR-29b

The 293T cells were co-transfected with the VEGFA-3'UTR-WT plasmid and the miR$29 \mathrm{~b}$ mimics plasmid; compared with the VEGFA- $3^{\prime} \mathrm{UTR}-\mathrm{WT}+\mathrm{NC}$, the average activity ratio of firefly luciferase and renilla luciferase $(\mathrm{Y} / \mathrm{H})$ in the VEGFA-3'UTR-WT + miR-29b group decreased $(P<0.05)$. However, there was no significant difference in the $\mathrm{Y} / \mathrm{H}$ value between 
Fig. 4. Verification of the targeting relationship between miR-29b and $V E G$ $F A$. Top, the binding site of miR-29b and VEGFA in 3'UTR; Bottom, the luciferase expression measured 48 hour after 293T cells were transfected with VEGFA-3'UTR-WT + miR-29b/ NC and VEGFA-3'UTR-MUT + miR-29b/NC; miR-29b, microRNA-29b; VEGFA, vascular endothelial growth factor $\mathrm{A}$; ${ }^{* *}, P<0.05$.

Fig. 5. The expression of miR-29b and mRNA expressions of VEGFA, ERK, Akt, $m$ TOR and $B c l-2$ in each group. (a) expression of miR-29b and mRNA expressions of VEGFA, ERK, Akt, mTOR and Bcl-2 in nude mice inoculated with RL-95-2 cells; (b) expression of miR-29b and mRNA expressions of VEGFA, ERK, $A k t, m T O R$ and $B c l-2$ in nude mice inoculated with HEC1-B cells; miR-29b, microRNA-29b; VEGFA, vascular endothelial growth factor A; ERK, extracellular regulated protein kinases; Akt, protein kinase B; mTOR, mammalian target of rapamycin; Bcl-2, B-cell lymphoma-2; *, $P<0.05$ in comparison to the normal group; \#, $P<$ 0.05 in comparison to the blank group.
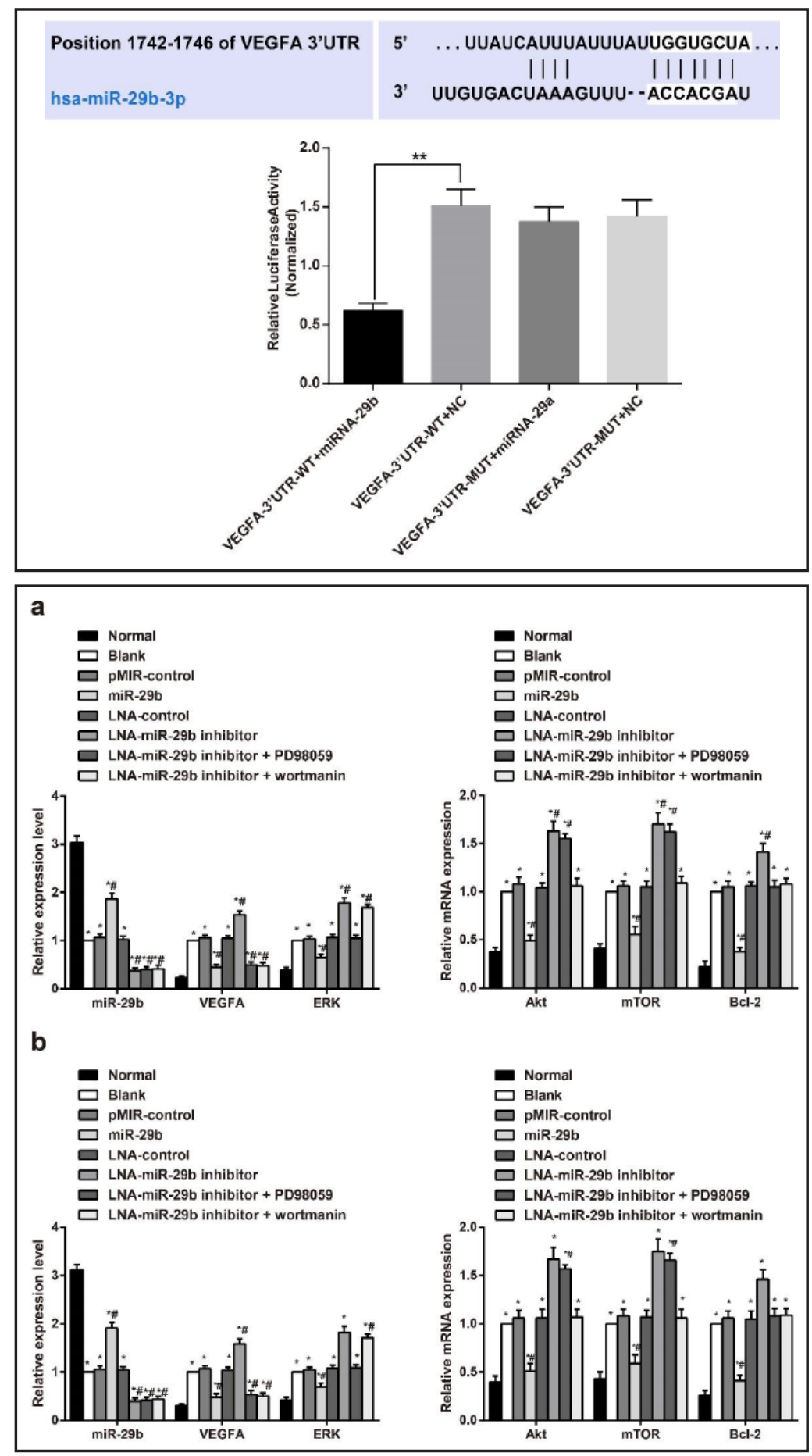

the VEGFA-3'UTR-MUT + NC and the VEGFA-3'UTR-MUT + miR-29b groups $(P>0.05)$ (Fig. 4).

\section{Successful transfection of miR-29b and LNA-miR-29b inhibitors plasmids}

The recombination expression vectors, pMIR-miR-29b (miR-29b plasmid) and empty vector, were used to transfect RL-95-2 and HEC-1-B cells, respectively. Green fluorescent protein (GFP) expression was determined using a fluorescence microscope. The results demonstrated that cells in each group all presented green light, suggesting successful transfection. Afterward, the transfection was repeated until the titer reached $10^{8} \mathrm{pfu} / \mathrm{ml}$. 


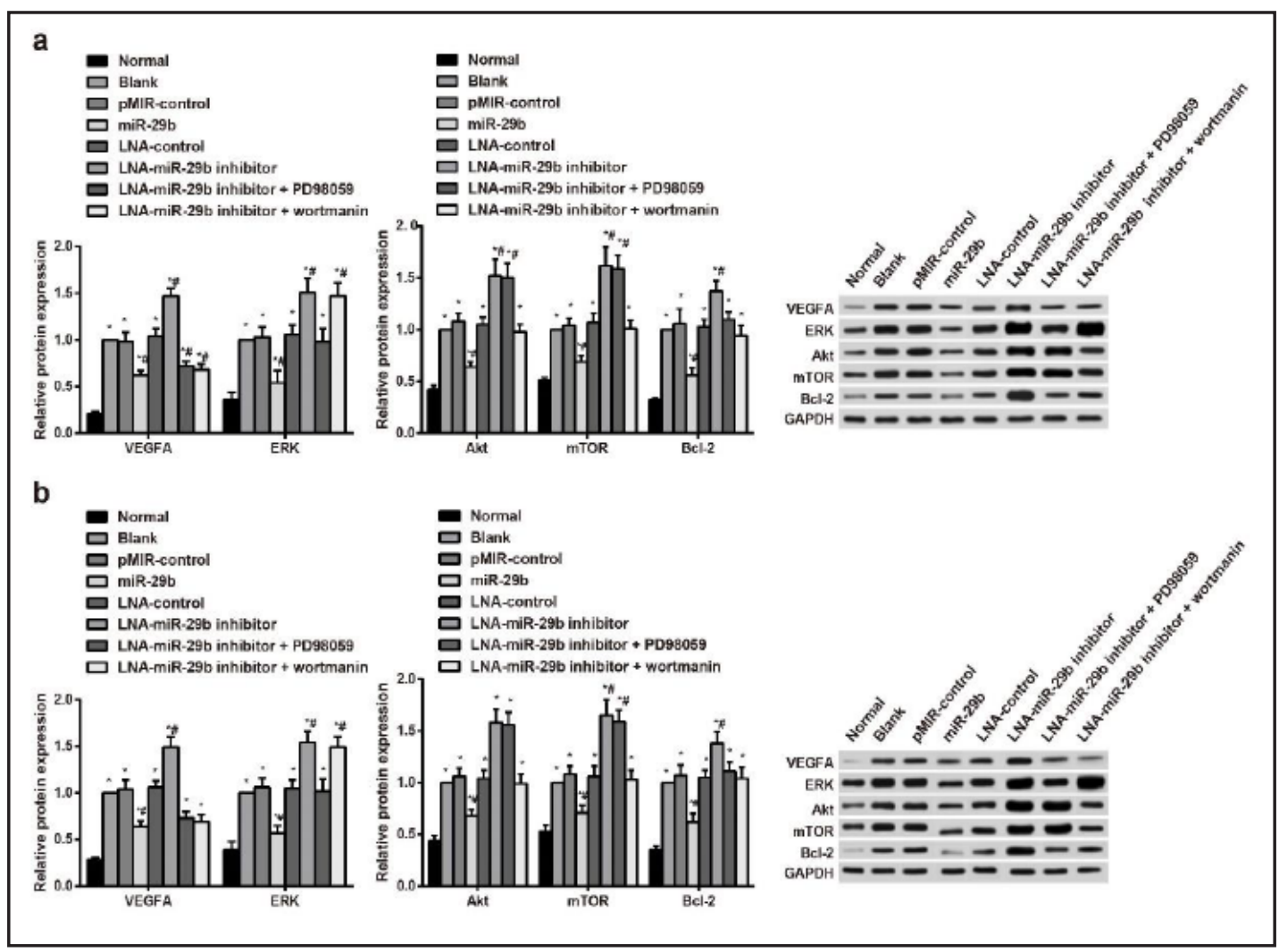

Fig. 6.Protein expressions of VEGFA, ERK, Akt, mTOR and Bcl-2 in each group. (a) Protein expressions of VEGFA, ERK, Akt, mTOR and Bcl-2 in nude mice inoculated with RL-95-2 cells; (b) Protein expressions of VEGFA, ERK, Akt, mTOR and Bcl-2 in nude mice inoculated with HEC-1-B cells; miR-29b, microRNA-29b; VEGFA, vascular endothelial growth factor A; ERK, extracellular regulated protein kinases; Akt, protein kinase B; mTOR, mammalian target of rapamycin; Bcl-2, B-cell lymphoma-2; *, $P<0.05$ in comparison to the normal group; \#, $P<0.05$ in comparison to the blank group.

Comparisons of $m R N A$ and protein expressions of miR-29b, VEGFA, ERK, Akt, mTOR and $\mathrm{Bcl}-2$ in each group

There were no significant differences in the expressions of miR-29b, VEGFA, ERK, Akt, mTOR and Bcl-2 between the RL-95-2 and HEC-1-B cells (all $P>0.05$ ). In addition, compared with the normal group, miR-29b expression was down-regulated, while the mRNA expressions of VEGFA, ERK, Akt, mTOR and Bcl-2 were up-regulated in the other groups (all $P<0.05)$. No significant differences were found among the blank, pMIR-control and LNAcontrol groups (all $P>0.05$ ). Compared with the blank group, miR-29b expression was increased, while the mRNA expressions of VEGFA, ERK, Akt, mTOR and Bcl-2 were decreased in the pMIR-miR-29b group. The LNA-miR-29b inhibitors group exhibited elevated miR-29b expression and decreased the mRNA expressions of VEGFA, ERK, Akt, mTOR and Bcl-2 (all $P<0.05)$. Additionally, miR-29b expression was reduced in the LNA-miR-29b inhibitors + PD98059 and LNA-miR-29b inhibitors + wortmannin groups (all $P<0.05$ ) (Fig. 5). Compared with the normal group, the protein expressions of VEGFA, ERK, Akt, mTOR and Bcl-2 of other groups were increased (all $P<0.05$ ). However, there were no significant differences among the blank, pMIR-control and LNA-control groups (all $P>0.05$ ). The protein expressions of VEGFA, ERK, Akt, mTOR and Bcl-2 were lower in the pMIR-miR-29b group but higher in the LNA-miR-29b inhibitors group than in the blank group (all $P<0.05$ ) (Fig. 6), suggesting that miR-29b could inhibit the progression of EC by down-regulating the MAPK/ERK and PI3K/ Akt signaling pathways. 


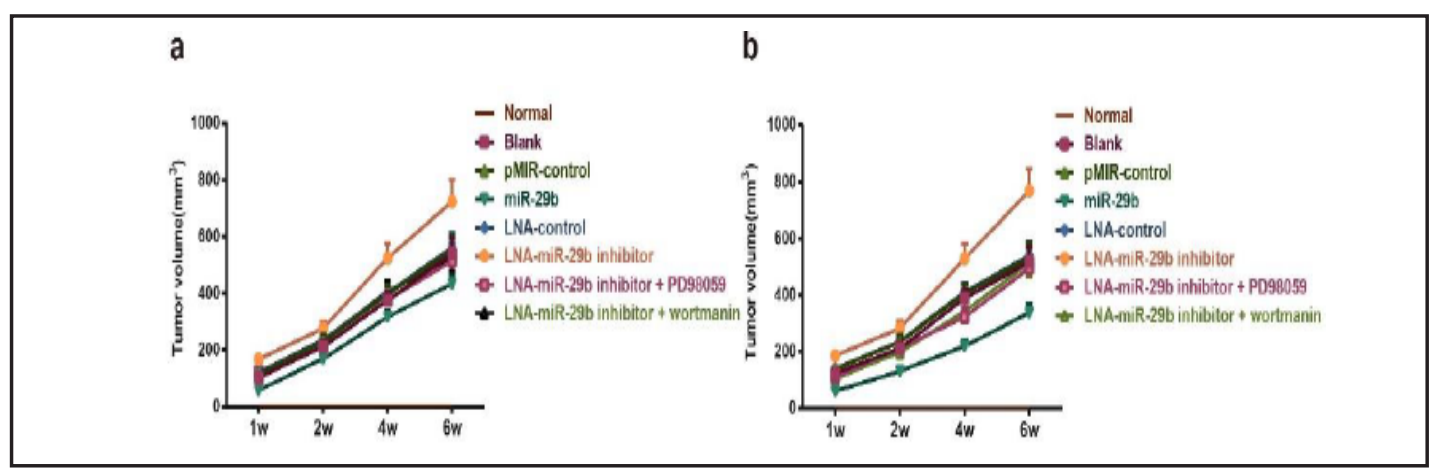

Fig. 7. Tumor growth curves of the nude mice with tumor xenografts. (A), Tumor growth curves of nude mice inoculated with RL-95-2 cells; (B), Tumor growth curves of nude mice inoculated with HEC-1-B cells.

Comparison of tumor weight, tumor growth inhibitory rate and tumor metastasis inhibitory rate of nude mice in each group

On the $4^{\text {th }}$ day of the tumor inoculation, a gradually increased tumor volume was observed in the blank, pMIR-control, pMIR-miR-29b, LNA-control, LNA-miR-29b inhibitors, LNA-miR-29b inhibitors + PD98059 and LNA-miR-29b inhibitors + wortmannin groups, with a growing nodule appearing in the partial tumor. For the nude mice inoculated with the RL-95-2 and HEC-1-B cells between 1 to 6 weeks, a significantly increased tumor growth was found in the LNA-miR-29b inhibitors group compared to the other groups, and the tumor growth was the lowest in the pMIR-miR-29b group (Fig. 7). When the mice were dissected, compared with the blank group, the tumor weight in the LNA-miR-29b inhibitors + PD98059 and LNA-miR-29b inhibitors + wortmannin groups was slightly decreased, and the tumor was the lightest in the pMIR-miR-29b group. The tumor control rate and tumor metastasis inhibitory rate were significantly increased in the LNA-miR-29b inhibitors + PD98059 and LNA-miR-29b inhibitors + wortmannin groups compared with the pMIR-miR-29b group $(P$ $<0.05$ ) (Table 5 and Table 6). These results indicated that the overexpression of miR-29b could inhibit the growth and metastasis of xenograft tumors in nude mice.

Comparison of MVD expression of nude mice in each group

The CD34 positive staining was restricted to the cell membrane and cytoplasm of the vascular endothelial cell to form a strip or ring. The CD34 positive cells were few, and angiogenesis was not obvious in the normal group. The number of CD34 positive cells and angiogenesis were the highest in the LNA-miR-29b inhibitors group, followed by the blank, pMIR-control, LNA-control, LNA-miR-29b inhibitors + PD98059 and LNA-miR-29b inhibitors + wortmannin groups, and the least in the pMIR-miR-29b group. The CD34 staining showed that single brown cells or cell clusters, which were markedly different from the background staining, were considered as one microvessel. No significant difference in MVD expression was found between the nude mice inoculated with the RL-95-2 and the HEC-1-B cells $(P>$ $0.05)$. Compared with the normal group, MVD expression was elevated in the other groups $(P<0.05)$. There were no significant differences in MVD expression among the blank, pMIRcontrol, LNA-control, LNA-miR-29b inhibitors + PD98059 and LNA-miR-29b inhibitors + wortmannin groups $(P>0.05)$. Compared with the blank group, MVD expression was decreased in the pMIR-miR-29b group but increased in the LNA-miR-29b inhibitors group $(P<0.05)$ (Table 7), suggesting that miR-29b could inhibit angiogenesis in EC, thereby suppressing the progression of EC.

\section{Discussion}

A recent study has demonstrated that angiogenesis, the process of new blood-vessel growth, has been implicated in tumor growth in the endometrium [22]. In this study, we explored whether miR-29b can regulate the MAPK/ERK and PI3K/Akt signaling pathways 
Table 5. Comparisons of tumor weight, tumor growth inhibitory rate and tumor metastasis inhibitory rate in nude mice inoculated with RL-95-2 cells in each group ( $\overline{\mathrm{x}} \pm \mathrm{s}$ ). Notes: EC, endometrial carcinoma; ${ }^{*}, P<$ 0.05 in comparison to the blank group

\begin{tabular}{lclll}
\hline Group & $\mathrm{n}$ & $\begin{array}{l}\text { Tumor weight } \\
(\mathrm{g})\end{array}$ & $\begin{array}{l}\text { Tumor growth inhibitory rate } \\
(\%)\end{array}$ & $\begin{array}{l}\text { Tumor metastasis inhibitory rate } \\
(\%)\end{array}$ \\
\hline Normal & 10 & & & \\
Blank & 10 & $8.916 \pm 0.472$ & & \\
pMIR-control & 10 & $8.683 \pm 0.463$ & & $19.35 \pm 1.91$ \\
pMIR-miR-29b & 10 & $2.149 \pm 0.221^{*}$ & $18.03 \pm 1.77$ & \\
LNA-control & 10 & $8.802 \pm 0.460$ & & $31.91 \pm 2.99$ \\
LNA-miR-29b inhibitors & 10 & $13.216 \pm 0.527^{*}$ & & $28.73 \pm 2.8$ \\
LNA-miR-29b inhibitors + & 10 & $7.593 \pm 0.325^{*}$ & $26.33 \pm 2.56$ & \\
$\begin{array}{l}\text { PD98059 } \\
\text { LNA-miR-29b inhibitors + }\end{array}$ & 10 & $7.776 \pm 0.338^{*}$ & $29.18 \pm 2.71$ & \\
wortmannin & & & & \\
\hline
\end{tabular}

Table 6. Comparisons of tumor weight, tumor growth inhibitory rate and tumor metastasis inhibitory rate in nude mice inoculated with HEC-1-B cells in each group ( $\bar{x} \pm$ s). Notes: EC, endometrial carcinoma; ${ }^{*}, P<$ 0.05 in comparison to the blank group

\begin{tabular}{|c|c|c|c|c|}
\hline Group & $\mathrm{n}$ & $\begin{array}{l}\text { Tumor weight } \\
\text { (g) }\end{array}$ & $\begin{array}{l}\text { Tumor growth inhibitory } \\
\text { rate }(\%)\end{array}$ & $\begin{array}{l}\text { Tumor metastasis inhibitory } \\
\text { rate }(\%)\end{array}$ \\
\hline Normal & 10 & & & \\
\hline Blank & 10 & $8.876 \pm 0.467$ & & \\
\hline pMIR-control & 10 & $8.612 \pm 0.483$ & & \\
\hline pMIR-miR-29b & 10 & $2.125 \pm 0.211^{*}$ & $16.87 \pm 1.67$ & $18.94 \pm 1.91$ \\
\hline LNA-control & 10 & $8.713 \pm 0.471$ & & \\
\hline LNA-miR-29b inhibitors & 10 & $\begin{array}{l}13.172 \pm \\
0.547^{*}\end{array}$ & & \\
\hline $\begin{array}{l}\text { LNA-miR-29b inhibitors + } \\
\text { PD98059 }\end{array}$ & 10 & $7.217 \pm 0.335^{*}$ & $25.87 \pm 2.56$ & $27.89 \pm 2.79$ \\
\hline $\begin{array}{l}\text { LNA-miR-29b inhibitors + } \\
\text { wortmannin }\end{array}$ & 10 & $7.625 \pm 0.348^{*}$ & $27.29 \pm 2.61$ & $30.36 \pm 2.83$ \\
\hline
\end{tabular}

Table 7. Comparison of MVD expression of nude mice inoculated with RL-95-2 and HEC-1-B cells in each group ( $\overline{\mathrm{x}} \pm \mathrm{s}$ ). Notes: EC, endometrial carcinoma; MVD, microvessel density; ${ }^{*}, P<0.05$ in comparison to the normal group; \#, $P<0.05$ in comparison to the blank group

\begin{tabular}{lcll}
\hline Group & $\mathrm{n}$ & $\begin{array}{c}\text { MVD expression } \\
\text { in RL-95-2 cells }\end{array}$ & $\begin{array}{c}\text { MVD expression } \\
\text { in HEC-1-B cells }\end{array}$ \\
\hline Normal & 10 & $6.27 \pm 0.62$ & $6.35 \pm 0.63$ \\
Blank & 10 & $25.2 \pm 2.20^{*}$ & $27.76 \pm 1.97^{*}$ \\
pMIR-control & 10 & $26.54 \pm 2.03^{*}$ & $26.07 \pm 2.57^{*}$ \\
pMIR-miR-29b & 10 & $14.32 \pm 1.44^{*} \#$ & $14.56 \pm 1.43^{*} \#$ \\
LNA-control & 10 & $26.01 \pm 2.12^{*}$ & $26.28 \pm 2.34^{*}$ \\
LNA-miR-29b inhibitors & 10 & $36.17 \pm 2.4^{*} \#$ & $36.86 \pm 2.15^{*} \#$ \\
LNA-miR-29b inhibitors + PD98059 & 10 & $24.03 \pm 1.65^{*} \#$ & $24.97 \pm 1.64^{*} \#$ \\
LNA-miR-29b inhibitors + wortmannin & 10 & $24.69 \pm 1.63^{*} \#$ & $25.56 \pm 1.63^{*} \#$ \\
\hline
\end{tabular}

to affect angiogenesis in EC by targeting VEGFA. Collectively, our study demonstrated that miR-29b negatively modulates the MAPK/ERK and PI3K/Akt signaling pathways to inhibit angiogenesis in EC by targeting VEGFA.

Initially, compared with the adjacent normal tissues, miR-29b expression was significantly down-regulated in the EC tissues. To the best of our knowledge, miR-29b can act as a tumor suppressor to inhibit the growth of cancer cells. In our study, using cell transfections with different plasmids, the results revealed that miR-29b could inhibit cell invasion and metastasis in both the EC RL-95-2 and the HEC-1-B cell lines. According to a study by Hiroki et al., miR-29b exhibited low expression in endometrial serous adenocarcinoma, and the reduction of miR-29b was correlated with a high degree of vascular invasion and poor overall survival, suggesting that the low expression of miR-29b can serve as an indicator of a poor prognosis of endometrial serous adenocarcinoma [23]. And Teng et al. demonstrates that miR-29b has a negative correlation with DNA methyltransferase (DNMT) 3A/3B expression in patients with epithelial ovarian cancer, which may provide a novel therapeutic 
option for the treatment of epithelial ovarian cancer [24]. A previous study also clarifies that miR-29b plays a significant role in the mouse early embryonic development through modulating the expressions of DNMT3A and DNMT3B [25]. Base on the study of Alowayed et al., LEFTY2 can negatively regulate the endometrial cell proliferation and migration through inhibiting FAK activity and MKi67 expression, as well as elevating miR-200a and E-cadherin [26]. Additionally, Qiang et al. have provided evidence that the down-regulation of miR$29 \mathrm{~b}$ is one of the mechanisms underlying the excessive accumulation of the extracellular matrix, which is the most notable pathophysiological characteristic of uterine leiomyoma [27]. Interestingly, our findings indicated that, compared with the adjacent normal tissues, VEGFA mRNA and protein expressions were up-regulated in the EC tissues. In addition, VEGFA mRNA and protein expressions were down-regulated in the pMIR-miR-29b group and up-regulated in the LNA- miR-29b group, suggesting the negative targeting relationship between miR-29b and VEGFA. Our dual-luciferase reporter system indicated that VEGFA was proven to be a target gene of miR-29b and miR-29 negatively regulated VEGFA. Recent studies noted that the growth factor VEGFA was identified as a target gene of miR-29b, which was consistent with our study [28, 29]. According to Liu et al., miR-29b targeted VEGFA and could be used as novel biomarkers in diagnosis of glioma [30]. Of the angiogenic factors, VEGFA is a dimeric glycoprotein that plays a crucial role in promoting the proliferation and migration of endothelial cells and increasing the permeability of tumor-related blood vessels through binding the tyrosine kinase receptors flt-1 (VEGFR-1) and KDR (VEGFR-2) [31]. Largely consistent with our results, Aghajanian et al. advocated the positive association between high circulating VEGF-A levels with the poor outcome of EC patients [32]. IHC discovered that increased MVD expression was found in the EC tissues compared with the adjacent normal tissues. Angiogenesis is important for the continuous growth of tumors and the development of metastases in EC, and MVD has been commonly used to measure tumor-related angiogenesis [33]. A previous study demonstrated that the down-regulation of miR-29b could lead to angiogenesis, metastasis and invasion of hepatocellular carcinoma (HCC) [34]. In comparison to normal nude mice, those with a tumor xenograft had increased MVD. The nude mice in the pMIR-miR-29b group had decreased MVD, and those in the LNAmiR-29b inhibitors group had increased MVD compared to those in the blank group, which suggested that miR-29b could inhibit angiogenesis.

Additionally, we found that, compared with the adjacent normal tissues, the mRNA and protein expressions of ERK, Akt, mTOR and Bcl-2 were up-regulated in the EC tissues. Compared with the blank group, ERK, Akt, mTOR and Bcl-2 mRNA and protein expressions were decreased in the pMIR-miR-29b group and increased in the LNA-miR-29b inhibitors group. As Holland et al. demonstrated, once VEGFA signaling is blocked by VEGF dominant negative receptors or neutralizing antibodies, tumor angiogenesis and proliferation are damaged [31]. Biroccio et al. also found that the overexpression of Bcl-2 enhanced the metastatic potential of human breast cancer cells [35]. In the study by Iervolino et al., overexpressed Bcl-2 was observed in human melanoma cells exposed to hypoxic conditions, and Bcl-2 could increase VEGF promoter activity by the hypoxia-inducible factor-1 (HIF-1) and promote HIF-1 DNA binding activity [36]. The activation of PI3K and the downstream serine-threonine protein kinase Akt and mTOR promote the expression of HIF-1 target gene under nonhypoxic conditions [37]. Intriguingly, Laughner et al., demonstrated that HIF-1a was up-regulated through activated PI3K and Akt in human prostate cancer cells [38]. Gille et al. reported that mTOR stimulation led to PI3K in main endothelial cells and promoted cell migration, and mTOR-selective VEGF could also induce angiogenesis [39]. In addition, Trisciuoglio et al. noted that MAPK- and PI3K-dependent pathways are proven to participate in the angiogenesis mediated by VEGF [37]. Thus, the up-regulation of miR-29b resulted in decreased VEGFA by negatively down-regulating the MAPK/ERK and PI3K/Akt signaling pathways.

Overall, we may conclude that miR-29b negatively modulates the MAPK/ERK and PI3K/ Akt signaling pathways to inhibit angiogenesis in EC by targeting VEGFA. Our results provide important novel information to further understand the therapeutic effects of low expression

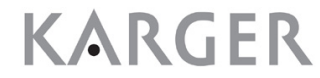


of VEGFA by restoring miR-29b in angiogenesis in EC. However, further work needs to address which of these possibilities apply and whether they all act in unison.

\section{Acknowledgments}

This work was supported by the Natural Science Foundation of Jiangxi Province (20142BAB215001), National Science Foundation Grants of China (81560395), the Science and Technology Planning Project of Health and Family Planning Commission of Jiangxi Province (No. 700766003), and the Youth Science Foundation of Jiangxi Provincial Department of Education (No. 00016798). We would like to acknowledge the helpful comments on this paper received from our reviewers.

\section{Disclosure Statement}

None.

\section{References}

1 Liao Y, Lu W, Che Q, Yang T, Qiu H, Zhang H, He X, Wang J, Qiu M, Zou Y, Gu W, Wan X: SHARP1 suppresses angiogenesis of endometrial cancer by decreasing hypoxia-inducible factor-1alpha level. PLoS One 2014;9:e99907.

2 Hill EK, Dizon DS: Medical therapy of endometrial cancer: current status and promising novel treatments. Drugs 2012;72:705-713.

- 3 Oza AM, Elit L, Tsao MS, Kamel-Reid S, Biagi J, Provencher DM, Gotlieb WH, Hoskins PJ, Ghatage P, Tonkin KS, Mackay HJ, Mazurka J, Sederias J, Ivy P, Dancey JE, Eisenhauer EA: Phase II study of temsirolimus in women with recurrent or metastatic endometrial cancer: a trial of the NCIC Clinical Trials Group. J Clin Oncol 2011;29:3278-3285.

4 Siegel R, Ma J, Zou Z, Jemal A: Cancer statistics, 2014. CA Cancer J Clin 2014;64:9-29.

-5 Vale CL, Tierney J, Bull SJ, Symonds PR: Chemotherapy for advanced, recurrent or metastatic endometrial carcinoma. Cochrane Database Syst Rev 2012;(8):CD003915.

6 Zhang J, Du YY, Lin YF, Chen YT, Yang L, Wang HJ, Ma D: The cell growth suppressor, mir-126, targets IRS-1. Biochem Biophys Res Commun 2008;377:136-140.

7 Luzi E, Marini F, Sala SC, Tognarini I, Galli G, Brandi ML: Osteogenic differentiation of human adipose tissuederived stem cells is modulated by the miR-26a targeting of the SMAD1 transcription factor. J Bone Miner Res 2008;23:287-295.

8 Panda H, Pelakh L, Chuang TD, Luo X, Bukulmez O, Chegini N: Endometrial miR-200c is altered during transformation into cancerous states and targets the expression of ZEBs, VEGFA, FLT1, IKKbeta, KLF9, and FBLN5. Reprod Sci 2012;19:786-796.

-9 Pan Y, Li C, Chen J, Zhang K, Chu X, Wang R, Chen L: The Emerging Roles of Long Noncoding RNA ROR (lincRNA-ROR) and its Possible Mechanisms in Human Cancers. Cell Physiol Biochem 2016;40:219-229.

10 Dykxhoorn DM: MicroRNAs and metastasis: little RNAs go a long way. Cancer Res 2010;70:6401-6406.

11 Fabian MR, Sonenberg N, Filipowicz W: Regulation of mRNA translation and stability by microRNAs. Annu Rev Biochem 2010;79:351-379.

12 Brabletz S, Brabletz T: The ZEB/miR-200 feedback loop--a motor of cellular plasticity in development and cancer? EMBO Rep 2010;11:670-677.

13 Guo Y, Lang X, Lu Z, Wang J, Li T, Liao Y, Jia C, Zhao W, Fang H: MiR-10b Directly Targets ZEB1 and PIK3CA to Curb Adenomyotic Epithelial Cell Invasiveness via Upregulation of E-Cadherin and Inhibition of Akt Phosphorylation. Cell Physiol Biochem 2015;35:2169-2180.

14 Langsch S, Baumgartner U, Haemmig S, Schlup C, Schafer SC, Berezowska S, Rieger G, Dorn P, Tschan MP, Vassella E: miR-29b Mediates NF-kappaB Signaling in KRAS-Induced Non-Small Cell Lung Cancers. Cancer Res DOI:10.1158/0008-5472.CAN-15-2580.

15 Mott JL, Kurita S, Cazanave SC, Bronk SF, Werneburg NW, Fernandez-Zapico ME: Transcriptional suppression of mir-29b-1/mir-29a promoter by c-Myc, hedgehog, and NF-kappaB. J Cell Biochem 2010;110:1155-1164. 


\section{Cellular Physiology Cell Physiol Biochem 2017;41:933-946

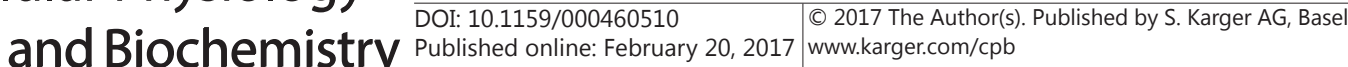 \\ Chen et al.: MiR-29b Inhibits Angiogenesis in EC}

16 Dai N, Zhong ZY, Cun YP, Qing Y, Chen C, Jiang P, Li MX, Wang D: Alteration of the microRNA expression profile in human osteosarcoma cells transfected with APE1 siRNA. Neoplasma 2013;60:384-394.

17 Garzon R, Heaphy CE, Havelange V, Fabbri M, Volinia S, Tsao T, Zanesi N, Kornblau SM, Marcucci G, Calin GA, Andreeff M, Croce CM: MicroRNA 29b functions in acute myeloid leukemia. Blood 2009;114:5331-5341.

$>18$ Zhang K, Zhang C, Liu L, Zhou J: A key role of microRNA-29b in suppression of osteosarcoma cell proliferation and migration via modulation of VEGF. Int J Clin Exp Pathol 2014;7:5701-5708.

19 Baeriswyl V, Christofori G: The angiogenic switch in carcinogenesis. Semin Cancer Biol 2009;19:329-337.

20 Lin SL, Yan LY, Zhang XT, Yuan J, Li M, Qiao J, Wang ZY, Sun QY: ER-alpha36, a variant of ER-alpha, promotes tamoxifen agonist action in endometrial cancer cells via the MAPK/ERK and PI3K/Akt pathways. PLoS One 2010;5:e9013.

21 Adanu RM: IJGO at the FIGO 2015 Congress. Int J Gynaecol Obstet 2015;131:1-2.

22 Saito M, Sato Y, Watanabe J, Kuramoto H, Kaba S, Fukuda T: Angiogenic factors in normal endometrium and endometrial adenocarcinoma. Pathol Int 2007;57:140-147.

-23 Hiroki E, Akahira J, Suzuki F, Nagase S, Ito K, Suzuki T, Sasano H, Yaegashi N: Changes in microRNA expression levels correlate with clinicopathological features and prognoses in endometrial serous adenocarcinomas. Cancer Sci 2010;101:241-249.

24 Teng Y, Zuo X, Hou M, Zhang Y, Li C, Luo W, Li X: A Double-Negative Feedback Interaction between MicroRNA-29b and DNMT3A/3B Contributes to Ovarian Cancer Progression. Cell Physiol Biochem 2016;39:2341-2352.

-25 Zhang J, Wang Y, Liu X, Jiang S, Zhao C, Shen R, Guo X, Ling X, Liu C: Expression and potential role of microRNA-29b in mouse early embryo development. Cell Physiol Biochem 2015;35:1178-1187.

26 Alowayed N, Salker MS, Zeng N, Singh Y, Lang F: LEFTY2 Controls Migration of Human Endometrial Cancer Cells via Focal Adhesion Kinase Activity (FAK) and miRNA-200a. Cell Physiol Biochem 2016;39:815-826.

27 Qiang W, Liu Z, Serna VA, Druschitz SA, Liu Y, Espona-Fiedler M, Wei JJ, Kurita T: Down-regulation of miR$29 \mathrm{~b}$ is essential for pathogenesis of uterine leiomyoma. Endocrinology 2014;155:663-669.

28 Kwiecinski M, Elfimova N, Noetel A, Tox U, Steffen HM, Hacker U, Nischt R, Dienes HP, Odenthal M: Expression of platelet-derived growth factor-C and insulin-like growth factor I in hepatic stellate cells is inhibited by miR-29. Lab Invest 2012;92:978-987.

29 Santa LM, Teshima LY, Forero JV, Giraldo AO: AngiomiRs: Potential Biomarkers of Pregnancy's Vascular Pathologies. J Pregnancy 2015;2015:320386.

-30 Liu Q Liao F, Wu H, Cai T, Yang L, Fang J: Different expression of miR-29b and VEGFA in glioma. Artif Cells Nanomed Biotechnol 2016;44:1927-1932.

-31 Holland CM, Day K, Evans A, Smith SK: Expression of the VEGF and angiopoietin genes in endometrial atypical hyperplasia and endometrial cancer. Br J Cancer 2003;89:891-898.

-32 Aghajanian C, Sill MW, Darcy KM, Greer B, McMeekin DS, Rose PG, Rotmensch J, Barnes MN, Hanjani P, Leslie KK: Phase II trial of bevacizumab in recurrent or persistent endometrial cancer: a Gynecologic Oncology Group study. J Clin Oncol 2011;29:2259-2265.

-33 Stefansson IM, Salvesen HB, Akslen LA: Vascular proliferation is important for clinical progress of endometrial cancer. Cancer Res 2006;66:3303-3309.

-34 Fang JH, Zhou HC, Zeng C, Yang J, Liu Y, Huang X, Zhang JP, Guan XY, Zhuang SM: MicroRNA-29b suppresses tumor angiogenesis, invasion, and metastasis by regulating matrix metalloproteinase 2 expression. Hepatology 2011;54:1729-1740.

35 Biroccio A, Candiloro A, Mottolese M, Sapora O, Albini A, Zupi G, Del Bufalo D: Bcl-2 overexpression and hypoxia synergistically act to modulate vascular endothelial growth factor expression and in vivo angiogenesis in a breast carcinoma line. FASEB J 2000;14:652-660.

-36 Iervolino A, Trisciuoglio D, Ribatti D, Candiloro A, Biroccio A, Zupi G, Del Bufalo D: Bcl-2 overexpression in human melanoma cells increases angiogenesis through VEGF mRNA stabilization and HIF-1-mediated transcriptional activity. FASEB J 2002;16:1453-1455.

-37 Trisciuoglio D, Iervolino A, Zupi G, Del Bufalo D: Involvement of PI3K and MAPK signaling in bcl-2-induced vascular endothelial growth factor expression in melanoma cells. Mol Biol Cell 2005;16:4153-4162.

-38 Laughner E, Taghavi P, Chiles K, Mahon PC, Semenza GL: HER2 (neu) signaling increases the rate of hypoxia-inducible factor 1alpha (HIF-1alpha) synthesis: novel mechanism for HIF-1-mediated vascular endothelial growth factor expression. Mol Cell Biol 2001;21:3995-4004.

-39 Gille H, Kowalski J, Li B, LeCouter J, Moffat B, Zioncheck TF, Pelletier N, Ferrara N: Analysis of biological effects and signaling properties of Flt-1 (VEGFR-1) and KDR (VEGFR-2). A reassessment using novel receptor-specific vascular endothelial growth factor mutants. J Biol Chem 2001;276:3222-3230. 